\title{
Al and Machine Learning for Multi-spectral Imaging Applications
}

\author{
Dalila B. Megherbi, PhD \\ Director, Computer Machine/Human Intelligence Networking and Distributed Systems (CMINDS) Research Center \\ Faculty of Electrical and Computer Engineering, Francis College of Engineering \\ University of Massachusetts Lowell, USA
}

Multispectral sensing is of major importance for supporting many applications, such as bio-imaging and medical field. These days, Deep Learning AI techniques are, in many cases, used on a virtually empirical basis without the needed understanding of their performance and behavior. In particular, current deep learning techniques for multispectral machine vision are limited. Adding different layers and increasing the size of training data, (big data) will not be enough to shed light on these learning complexities. While various multi-spectral recognition algorithms have been proposed in the literature, unfortunately many of them remain in their infancy. This is mainly due to their lack of high recognition rates in the presence of time-varying image artifacts and conditions, even slight ones. In order to develop more accurate recognition systems, there is a primary need to identify and derive some of the causes that affect some multispectral target recognition accuracy rates. In this talk, I will focus on some of these causes and present our latest findings on investigation and analysis of how and what factors may affect the recognition accuracy rate of some of the most popular and currently widely used deep-learning algorithm for automatic multispectral object recognition and other applications. In particular, in this presentation, as an example, we propose and show how a "Fully" Convolutional Neural Network (FCNN)-based deep learning system with a novel Pixel Rearrangement technique results in significantly reduced computational complexity and improved accuracy than its state-of-the-art counterparts. We-present a benchmark comparison result with various existing approaches to highlight our reduced computational complexity but improved accuracy. 